\title{
Short communication: Shedding of Mycoplasma bovis and antibody responses in cows recently diagnosed with clinical infection
}

\author{
M. S. Hazelton, ${ }^{* 1}$ P. A. Sheehy, ${ }^{*}$ K. L. Bosward, ${ }^{*}$ A. M. Parker, ${ }^{*}$ J. M. Morton,† C. J. Dwyer,ł P. G. Niven,§ \\ and J. K. House* \\ ${ }^{*}$ Faculty of Science, Sydney School of Veterinary Science, The University of Sydney, Camden, New South Wales 2570, Australia \\ †Jemora Pty Ltd., Geelong, Victoria 3220, Australia \\ ¥Smithton Veterinary Service, Tasmania 7330, Australia \\ §Pure Source Dairy Farm Company Ltd., Qihe County, Dezhou City, Shandong Province 251100, China
}

\section{ABSTRACT}

Mycoplasma bovis can have significant consequences when introduced into immunologically naïve dairy herds. Subclinically infected carrier animals are the most common way that $M$. bovis is introduced into herds. Although $M$. bovis udder infections can be detected by milk sampling lactating animals before their introduction, currently, no definitive way of identifying $M$. bovis carrier animals that are nonlactating (i.e., calves, heifers, dry cows, or bulls) is available. Understanding the prevalence of $M$. bovis shedding from various body sites in clinically infected animals could inform strategies for the detection of subclinical infection in nonlactating stock. The mucosal surfaces of the nose, eye, and vagina of 16 cows with recent clinical mastitis caused by $M$. bovis were examined for the presence of $M$. bovis shedding. Blood was collected for serological evaluation by a commercially available ELISA. Mycoplasma bovis was isolated from the vagina of only $3(18.8 \%)$ of the cows and was not detected from the noses or eyes of any of the cows. Fifteen of the $16(93.8 \%)$ cows were seropositive to the ELISA. With such low prevalence of detection of $M$. bovis from the vagina and no detections from the noses or eyes of recently clinically infected animals, it is very likely that sampling these sites would be ineffective for detecting subclinical infection in cattle. Serology using the ELISA may have some use when screening animals for biosecurity risk assessment. However, more information regarding time to seroconversion, antibody longevity, and test diagnostic sensitivity and specificity are required to define the appropriate use of this ELISA for biosecurity purposes.

Key words: Mycoplasma, mastitis, shedding, dairy cow

Received July 17, 2017.

Accepted August 24, 2017.

${ }^{1}$ Corresponding author: mark.hazelton@sydney.edu.au

\section{Short Communication}

Mycoplasma bovis is one of the major pathogens of biosecurity significance to a dairy herd due to its highly contagious nature and unresponsiveness to most treatments (Fox, 2012). Generally, Mycoplasma species can cause disease at several different anatomical locations including the mammary gland, joints, respiratory (Pfützner and Sachse, 1996) and urogenital tracts (Ruhnke, 1994), eye (Alberti et al., 2006), and inner ear (Maunsell et al., 2012). Clinical diagnosis is typically based on clinical presentation with confirmation achieved via culture or PCR of milk, joint fluid, swabs, or tissues. Several molecular diagnostic assays (PCR, loop-mediated isothermal amplification) aimed at detecting various Mycoplasma spp. have been described in the veterinary literature. However, in Australia, most commercially available PCR assays are specific for detection of M. bovis.

In infected dairy herds, not all infected animals display clinical signs of disease. Understanding the prevalence of $M$. bovis shedding from various body sites is a logical first step in the development of any sampling and testing protocol designed for detection of subclinically infected animals. Little conclusive research is available on the prevalence of $M$. bovis shedding at different anatomical locations in clinically infected animals. Detecting carrier (i.e., subclinically infected) animals is difficult because there is no consistently infected body site to sample. Gonzalez et al. (1992) and Biddle et al. (2003) have highlighted the challenge in detecting subclinical mastitis due to intermittent shedding of $M$. bovis in milk. Furthermore, detecting subclinical infection in nonlactating stock poses an additional challenge.

Initial studies showed experimental inoculation of the pathogenic mycoplasma strain 01 (later identified as $M$. bovis) in the udder resulted in frequent but intermittent shedding of the same strain from the nose, eye, rectum, vagina, and urine of the inoculated cows (Jain et al., 1969). The same mycoplasma strain was also iso- 
lated from the lymph nodes, gastrointestinal contents, uterus, lung, liver, kidney, spleen, joint fluid, and fetus of the inoculated cows at postmortem. In a study by Jasper et al. (1974), following the recent diagnosis of mycoplasma mastitis in 6 cows, M. bovimastitidis (later reclassified as $M$. bovis) was isolated from the nose of 1 and vagina of 2 of these cows. Following the culling of these 6 cows, the remaining 112 cows in the herd were all culture negative for $M$. bovis in milk samples, but 1 cow returned an $M$. bovis positive nose swab, and 5 cows returned $M$. bovis positive vaginal swabs. A study by Biddle et al. (2005) demonstrated colonization of body sites other than the mammary gland, including the urogenital and respiratory tracts in 7 cows with Mycoplasma spp. udder infection that were sampled once weekly for $4 \mathrm{wk}$. However, prevalences of detection at each of the samplings were not reported and the nature of the mycoplasma infections (subclinical or clinical) was not described. Punyapornwithaya et al. (2010) investigated a herd experiencing clinical mastitis cases and isolated the same $M$. bovis strain from the mucosal surfaces of subclinical carriers in at least one nose, eye, ear, or vulvovaginal tract swab from $21 \%$ of the cows and $47 \%$ of the replacement stock sampled once over a 5-mo period commencing soon after the initial identification of clinical mastitis cases in the lactating cows. Three clinical cases (mastitis in 2 cows and polyarthritis in a calf) of $M$. bovis infections were diagnosed during the 5-mo period. Three subsequent samplings of each animal remaining within the herd over the following 10-mo period demonstrated a dramatic drop in $M$. bovis apparent prevalence to below $6.4 \%$ in both cohorts at each sampling occasion.

Dairy herds often expand or introduce new genetics by purchasing nonlactating stock (calves, heifers, dry cows, or bulls). As $M$. bovis can be detected from body sites other than the mammary gland, such purchases pose a risk for the introduction of $M$. bovis. More recently, studies have evaluated the use of ELISA to detect animals previously exposed to $M$. bovis (and hence, potentially infected; Wawegama et al., 2016).

Enhanced understanding of the anatomical locations of $M$. bovis infection and serological responses of clinically infected animals could help guide further development of sampling protocols aimed at increasing the sensitivity of detection of $M$. bovis in subclinically infected nonlactating stock. From this, an accurate assessment of biosecurity risk due to purchased stock could then be determined. Therefore, the objectives of this study were (1) to evaluate the frequency of $M$. bovis detection from readily accessible mucosal sites, and (2) to measure the serologic response to $M$. bovis, both in adult dairy cattle with recent clinical $M$. bovis mastitis.
During an $M$. bovis mastitis outbreak in a split calving (spring and autumn) dairy herd, 16 milking cows previously diagnosed with $M$. bovis mastitis via PCR (Thermo Scientific Pathoproof Major 4.2, Dairy Technical Services, Melbourne, Australia) were awaiting expiration of drug withhold periods before being sent for slaughter. These cows had been dried off and separated from the milking herd in the days before sampling for this study. The original milk samples used for $M$. bovis diagnosis had been collected $13 \mathrm{~d}$ before sampling for this study for 13 of the cows and $7 \mathrm{~d}$ prior for the other 3 cows. Swabs (15 cm rayon tip contained in Amies transport medium; FL Medical, Torreglia, Italy) were collected from the right nostril (nose), one conjunctival sac (eye), and the vagina of each cow, and blood was collected from the tail vein. The type of swab was selected for its affordability and accessibility and the sampling technique was designed for practical application in the field. The samples were chilled in a refrigerator and transported on ice until they reached the laboratory within 2 to $3 \mathrm{~d}$. The collection of all samples had the approval of the University of Sydney Animal Ethics Committee (protocol number 2013/6046).

The swab samples were cultured on selective medium made with Mycoplasma agar [Mycoplasma agar base (Oxoid CM0401, Waltham, MA); Milli-Q water; $0.2 \% \mathrm{wt} / \mathrm{vol}$ calf thymus DNA (Sigma D1501, Sigma, St. Louis, MO); Mycoplasma Selective Supplement G (Oxoid SR0059C); prepared by the Elizabeth Macarthur Agricultural Institute; New South Wales Department of Primary Industries, NSW, Australia]. Agar plates were then incubated at $37^{\circ} \mathrm{C}$ in candle jars with elevated $\mathrm{CO}_{2}$ levels for 5 to $10 \mathrm{~d}$. Following incubation, the plates were examined for colonies with gross morphology consistent with Mycoplasma species. For each isolate, several colonies were selected and aseptically transferred into $40 \mu \mathrm{L}$ of sterile PBS. The PBS suspension was vortexed and $10 \mu \mathrm{L}$ subcultured onto mycoplasma agar. Colonies from the subculture plates were subsequently harvested and stored in Cryobead tubes (Protect Multipurpose; Thermo Fisher Scientific, Waltham, MA) at $-80^{\circ} \mathrm{C}$. The residual $30 \mu \mathrm{L}$ of PBS was stored at $-20^{\circ} \mathrm{C}$ pending PCR evaluation to identify the species of Mycoplasma present.

Up to 3 PCR assays were used to determine the identity of the culture isolates. First, isolates were processed using a Mycoplasma spp. conventional PCR assay as described by Parker et al. (2017). This was followed by an Acholeplasma laidlawii conventional PCR assay designed using the Primer3 software program (Untergasser et al., 2012; Table 1). For each PCR assay, reaction mixtures contained $0.25 \mathrm{~m} M$ deoxynucleotide triphosphates, $2.5 \mathrm{mM} \mathrm{MgCl}$, $1.5 \mathrm{U}$ of GoTaq, $0.25 \mu M$ 
Table 1. Primers used for the Acholeplasma laidlawii conventional PCR and culture speciation

\begin{tabular}{lll}
\hline Name & Sequence & Target \\
\hline AlaiF & 5'-AACAAAGGGCACACAGTGGA-3' & $\begin{array}{l}\text { 16S-23S rRNA intergenic spacer region gene of Acholeplasma laidlawii } \\
\text { AlaiR }\end{array}$ \\
\hline
\end{tabular}

of each primer, $8 \mu \mathrm{L}$ of $5 \times$ buffer, and $5 \mu \mathrm{L}$ of DNA template in a final volume of $40 \mu \mathrm{L}$. Cycling conditions were $94^{\circ} \mathrm{C}$ for $5 \mathrm{~min}$, followed by 35 cycles of $94^{\circ} \mathrm{C}$ for $30 \mathrm{~s}, 55^{\circ} \mathrm{C}$ for $30 \mathrm{~s}, 72^{\circ} \mathrm{C}$ for $1 \mathrm{~min}$, and a final extension of $72^{\circ} \mathrm{C}$ for $5 \mathrm{~min}$. The assay was performed on a Bio-Rad-T100 Thermocycler (Bio-Rad Laboratories Pty Ltd., Gladesville, NSW, Australia). All PCR products amplified by the PCR assays underwent Sanger sequencing at the Australian Genome Research Facility Ltd. (Westmead, NSW, Australia) to validate species identification. Isolates that failed to amplify using the universal Mycoplasma spp. and A. laidlawii PCR assays were evaluated with a species-specific real-time probebased multiplex PCR assay as described by Parker et al. (2017), targeting M. bovis, Mycoplasma bovigenitalium, and Mycoplasma californicum. Reaction mixtures contained $0.5 \mathrm{~m} M$ deoxynucleotide triphosphates, 5 $\mathrm{m} M \mathrm{MgCl}_{2}, 0.5 \mathrm{U}$ of GoTaq polymerase, $1 \mu M$ each primer, $0.25 \mu \mathrm{M}$ probe, $2.0 \mu \mathrm{L}$ of $5 \times$ buffer, and $2 \mu \mathrm{L}$ of DNA template in a final volume of $10 \mu \mathrm{L}$. Cycling conditions were $95^{\circ} \mathrm{C}$ for $60 \mathrm{~s}$, followed by 40 cycles of $95^{\circ} \mathrm{C}$ for $30 \mathrm{~s}, 60^{\circ} \mathrm{C}$ for $30 \mathrm{~s}$, and $72^{\circ} \mathrm{C}$ for $30 \mathrm{~s}$. The assay was performed on a RotorGene 3000 RT-PCR System Thermocycler (Qiagen Pty Ltd., Chadstone Centre, VIC, Australia).

Sera were stored at $-80^{\circ} \mathrm{C}$ before serological evaluation using the Bio-X Mycoplasma bovis ELISA kit (BIO K 302, Bio-X Diagnostics, Jemelle, Belgium). The ELISA uses a recombinant protein from $M$. bovis expressed by Escherichia coli as a plate antigen. The assay was performed according to the manufacturer's instructions (http://www.biox.com/en/bio-k-302-monoscreen -abelisa-mycoplasma-bovis-indirect-monowell-p-250/). Briefly, $10 \mu \mathrm{L}$ of sera was diluted 1/100 using supplied dilution buffer before being added to individual wells with each sample analyzed in duplicate. Positive and negative control sera were run in triplicate. Two wells remained blank for the correction of background. Following incubation at $21^{\circ} \mathrm{C}$ for $1 \mathrm{~h}$, the plate was washed with the supplied washing solution. Conjugate solution (anti-mammalian IgG peroxidase-protein G horseradish peroxidase-labeled) was diluted 1:50 using the supplied dilution buffer and $100 \mu \mathrm{L}$ was then added to each well and incubated for $1 \mathrm{~h}$ at $21^{\circ} \mathrm{C}$. Following plate washing, $100 \mu \mathrm{L}$ of the supplied chromogen solution (tetramethylbenzidine) was added to each well before incubation for $10 \mathrm{~min}$ at $21^{\circ} \mathrm{C}$. The reaction was subsequently stopped using $50 \mu \mathrm{L}$ of the supplied stop solution (1 $M$ phosphoric acid), and the absorbance optical density (OD) of each well was read using a Polarstar Optima (450 nm) microplate reader (POLARstar Optima; BMG Labtech, Ortenberg, Germany). Sample OD was adjusted by subtracting the average OD from the blank wells. Each microplate was validated using the specified OD threshold for the positive and negative control sera. Optical density coefficient (ODC\%) was calculated as the proportion of a positive control and was calculated for each sample according to the following formula: (OD sample - OD negative serum)/ (OD positive serum - OD negative serum $) \times 100$. The standard deviation and coefficient of variation were calculated for each sample pair duplicate. Duplicate samples with a coefficient of variation greater than 0.2 and ODC $\%$ greater than 20 were rejected and the assay repeated. According to the manufacturer's directions, sera with an ODC\% equal to or greater than $37 \%$ were considered positive. An ODC\% less than 37\% was considered negative.

Mycoplasma spp. were detected in only 5 of the 16 cows and M. bovis was isolated from only 3 cows $(18.8 \%$; exact binomial $95 \% \mathrm{CI}=4.0$ to $45.6 \%$ ), all from the vagina (Table 2). Mycoplasma bovigenitalium was isolated from the vagina of 1 cow, Mycoplasma bovoculi was isolated from the eye of 2 cows, and a Mycoplasma spp. grown from the eye swab of 1 cow was unable to be speciated (Table 2).

Fifteen of the 16 cows were seropositive; thus, the sensitivity was $93.8 \%$ (exact binomial $95 \%$ CI 69.8 to $99.8 \%$ ) when using a recent prior positive result to the Thermo Scientific Pathoproof Major 4.2 M. bovis PCR in milk as the gold standard. Three of these seropositive cows had ODC\% values just above the ODC\% cut-off value of $37 \%$, at $40.6,44.1$, and $45.6 \%$ (Figure 1). One cow was seronegative with an ODC $\%$ of $26.3 \%$.

These results show that $M$. bovis is not highly likely to be isolated from a single swab from any of the nose, eye, or vagina of cows with recently diagnosed clinical $M$. bovis mastitis via PCR. This low prevalence of detection from other body sites in clinically infected mastitis cows is consistent with Jasper et al. (1974). Although Jain et al. (1969) reported more frequent isolation of $M$. bovis from the nose, eye, and vagina of 4 experimentally infected cows over time, detection was intermittent and inconsistent between cows. Biddle 


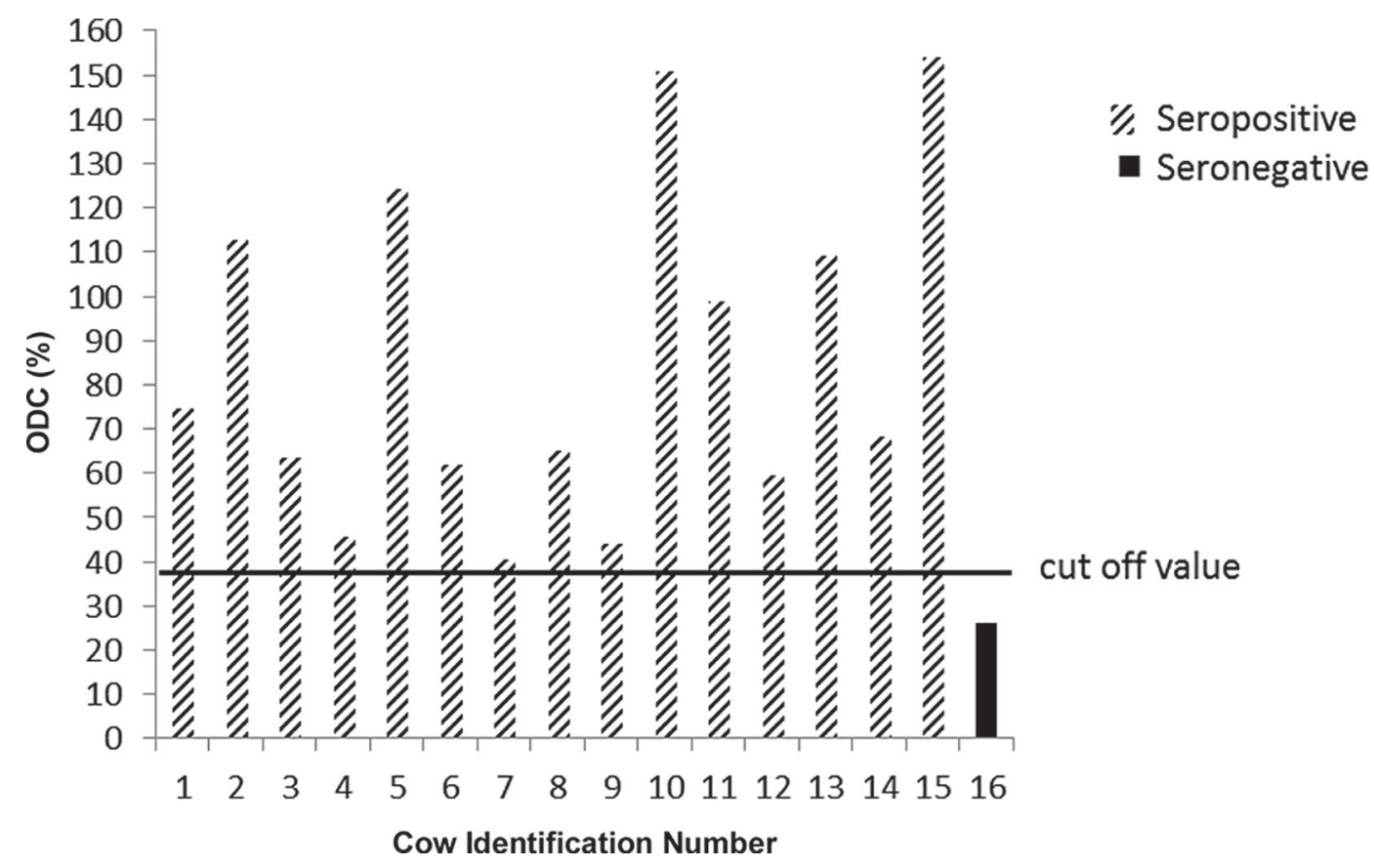

Figure 1. Bio-X Mycoplasma bovis ELISA (BIO K 302, Bio-X Diagnostics, Jemelle, Belgium) optical density coefficients (ODC\%) for the 16 cows.

et al. (2005) observed that colonization of accessible body sites other than the mammary gland was common in 7 cows recently identified to be infected (unknown if clinically or subclinically affected) with mycoplasma mastitis infections. However, the frequency of isolation of $M$. bovis at the different body sites at each sampling time point was not reported. Our failure to detect $M$. bovis from most sites from most cows could have been due, in part, to limitations of our sampling technique. Maunsell et al. (2012) identified colonization of the tonsils in calves after oral ingestion of infected colostrum. Although it is unknown how our study cows became infected, it is possible that longer guarded swabs rather than the $15 \mathrm{~cm}$ unguarded swabs we used may have enabled detection of increased numbers of infected animals due to the ability to sample the optimal site of

Table 2. Initial diagnostic (Thermo Scientific Pathoproof Major 4.2; Dairy Technical Services, Melbourne, Australia) cycle threshold for PCRpositive milk samples (PCR cycle threshold) from 16 cows with clinical mastitis and subsequent Mycoplasma spp. isolated from the noses, eyes, and vaginal swabs taken from these 16 cows 7 to $13 \mathrm{~d}$ after the PCR-positive milk sample was collected

\begin{tabular}{|c|c|c|c|c|}
\hline $\begin{array}{l}\text { Cow identification } \\
\text { number }\end{array}$ & $\begin{array}{l}\text { PCR cycle } \\
\text { threshold }\end{array}$ & \multicolumn{3}{|c|}{ Swab site } \\
\hline 1 & 16.7 & $-^{1}$ & - & - \\
\hline 2 & 31.7 & - & - & - \\
\hline 3 & 14.7 & - & - & - \\
\hline 4 & 36.3 & - & - & Mycoplasma bovigenitalium \\
\hline 5 & 17.4 & - & - & - \\
\hline 8 & 17.8 & - & M. bovoculi & - \\
\hline 9 & 20.4 & - & - & - \\
\hline 10 & 25.4 & - & - & - \\
\hline 11 & 25.2 & - & Unspeciated & M. bovis \\
\hline 12 & 32.5 & - & - & - \\
\hline 13 & 15.1 & - & - & M. bovis \\
\hline 14 & 36.2 & - & - & - \\
\hline
\end{tabular}


$M$. bovis colonization via the nose and vagina in adult cows. However, we deliberately used 15 -cm swabs because longer guarded swabs are less freely available and are more difficult to use. Swabbing tonsils of multiple adult cattle was likely to be impractical for routine field use due to imprecision in swab positioning and consequent risks to animal and human safety.

With such low prevalence of detection of $M$. bovis from the nose, eye, and vagina, it is reasonable to conclude that all clinically infected $M$. bovis mastitis cows do not equally experience detectable shedding at other body sites, at least 7 to $13 \mathrm{~d}$ after clinical mastitis diagnosis. Therefore, it is highly unlikely that $M$. bovis would be able to be detected at other body sites from all cows with a subclinical infection. Interestingly, Punyapornwithaya et al. (2010) detected a substantial proportion $(47 \%)$ of replacement stock with subclinical $M$. bovis colonization of the ear, eye, nose, or vulvovaginal tract soon after the start of a mastitis outbreak in the lactating cows in the same herd, but with 3 subsequent samplings of the same animals over the following $10 \mathrm{mo}$, prevalence of detection from these body sites declined to between 1.3 and $6.3 \%$. These findings suggest that timing of sampling in relation to a recent outbreak is important for detection of subclinical infections. These subsequent low prevalences of detection could be due to several reasons including clearance of the organism, intermittent shedding, or organism numbers being below the limit of detection.

When sampling stock pre-purchase to assess biosecurity risk, a single $M$. bovis positive nose, eye, or vaginal swab result from a cohort of animals could be considered evidence of increased biosecurity risk and the purchase of that particular cohort of animals avoided. Our detection of $M$. bovoculi and $M$. bovigentalium demonstrates the importance of having any isolates typed to determine their clinical significance; these species are of much lower clinical importance when compared with M. bovis (Fox et al., 2005). However, considering our findings and available research, culture of nose, eye, and vaginal swabs from nonlactating stock at a single point in time is likely to be ineffective for detecting subclinical $M$. bovis infection of individual animals.

Serologically, the Bio-X M. bovis ELISA (BIO K 302) had a diagnostic sensitivity of $93.8 \%$ in these recently clinically infected cows. Three of the 15 seropositive cows exhibited ODC $\%$ values $<46 \%$, thus approaching the manufacturer's cut-off value of $37 \%$ and one of the $M$. bovis PCR positive cows was seronegative. Immune responses to mycoplasma infection are poorly understood. The effects of colonization and antigenic stimulation on the onset, magnitude, and duration of antibody response for clinical and subclinical infections are poorly characterized and likely to vary between individual animals. Seroconversion is thought to take 2 to 3 wk (Nicholas et al., 2002; Wawegama et al., 2014), but the duration of elevated antibody titers post exposure has not been well characterized. Nicholas et al. (2002) detected high mean antibody titers 6 mo after vaccination in a group of calves, but antibody titers over time after natural $M$. bovis infections have not been reported. The timing of sampling relative to pathogen exposure is likely to be very important for interpretation. Further, longitudinal studies evaluating serial samples from experimentally or naturally infected animals may assist in interpretation of ELISA results. Although the serological response to mycoplasma exposure is poorly understood, there may still be utility in considering results collectively for a herd to determine a likely level of biosecurity risk by sampling a cohort of animals from the herd. For example, if all animals from a cohort are seronegative, this may indicate that recent $M$. bovis exposure of the herd is unlikely and hence the probability that any animals in the herd are currently infected is low. However, further studies are required to assess the herd level sensitivity when sampling various cohorts of animals from the herd. Detection of any seropositive animals may increase the level of suspicion that some animals in the herd are currently infected. However, the specificity of this ELISA and most appropriate ODC\% cut-off value are unknown, and a combination of herd history and further testing may be indicated to better evaluate biosecurity risk in this situation.

\section{ACKNOWLEDGMENTS}

This work was financially supported by Dairy Australia (Southbank, VIC, Australia). Technical assistance provided by Ann-Marie House and Karen Mathews from the Sydney School of Veterinary Science, Faculty of Science, The University of Sydney (Australia), was greatly appreciated.

\section{REFERENCES}

Alberti, A., M. F. Addis, B. Chessa, T. Cubeddu, M. Profiti, S. Rosati, A. Ruiu, and M. Pittau. 2006. Molecular and antigenic characterization of a Mycoplasma bovis strain causing an outbreak of infectious keratoconjunctivitis. J. Vet. Diagn. Invest. 18:41-51. https:// doi.org/10.1177/104063870601800106.

Biddle, M. K., L. K. Fox, M. A. Evans, and C. C. Gay. 2005. Pulsedfield gel electrophoresis patterns of Mycoplasma isolates from various body sites in dairy cattle with Mycoplasma mastitis. J. Am. Vet. Med. Assoc. 227:455-459. https://doi.org/10.2460/javma .2005.227.455.

Biddle, M. K., L. K. Fox, and D. D. Hancock. 2003. Patterns of mycoplasma shedding in the milk of dairy cows with intramammary mycoplasma infection. J. Am. Vet. Med. Assoc. 223:1163-1166. https://doi.org/10.2460/javma.2003.223.1163. 
Fox, L. K. 2012. Mycoplasma mastitis. Causes, transmission, and control. Vet. Clin. North Am. Food Anim. Pract. 28:225-237. https:// doi.org/10.1016/j.cvfa.2012.03.007.

Fox, L. K., J. H. Kirk, and A. Britten. 2005. Mycoplasma mastitis: A review of transmission and control. J. Vet. Med. Ser. B 52:153-160. https://doi.org/10.1111/j.1439-0450.2005.00845.x.

Gonzalez, R. N., R. A. Merrill, and P. A. Sears. 1992. Shedding of Mycoplasma bovis from the udder of naturally infected cows and its importance for the diagnosis of bovine intramammary infections. J. Dairy Sci. 75:259.

Jain, N. C., D. E. Jasper, and J. D. Dellinger. 1969. Experimental bovine mastitis due to mycoplasma. Cornell Vet. 59:10-28.

Jasper, D. E., J. M. Al-Aubaidi, and J. Fabricant. 1974. Epidemiologic observations on mycoplasma mastitis. Cornell Vet. 64:407-415.

Maunsell, F., M. B. Brown, J. Powe, J. Ivey, M. Woolard, W. Love, and J. W. Simecka. 2012. Oral inoculation of young dairy calves with Mycoplasma bovis results in colonization of tonsils, development of otitis media and local immunity. PLoS One 7:e44523. https://doi.org/10.1371/journal.pone.0044523.

Nicholas, R. A. J., R. D. Ayling, and L. P. Stipkovits. 2002. An experimental vaccine for calf pneumonia caused by Mycoplasma bovis: Clinical, cultural, serological and pathological findings. Vaccine 20:3569-3575. https://doi.org/10.1016/S0264-410X(02)00340-7.

Parker, A. M., J. K. House, M. S. Hazelton, K. L. Bosward, and P. A. Sheehy. 2017. Comparison of culture and a multiplex probe PCR for identifying Mycoplasma species in bovine milk, semen and swab samples. PLoS One 12:e0173422. https://doi.org/10.1371/journal .pone.0173422.
Pfützner, H., and K. Sachse. 1996. Mycoplasma bovis as an agent of mastitis, pneumonia, arthritis and genital disorders in cattle. Rev. Sci. Tech. 15:1477-1494.

Punyapornwithaya, V., L. K. Fox, D. D. Hancock, J. M. Gay, and J. R. Alldredge. 2010. Association between an outbreak strain causing Mycoplasma bovis mastitis and its asymptomatic carriage in the herd: A case study from Idaho, USA. Prev. Vet. Med. 93:66-70. https://doi.org/10.1016/j.prevetmed.2009.08.008.

Ruhnke, H. L. 1994. Mycoplasmas associated with bovine genital tract infections. Pages 56-61 in Mycoplasmosis in Animals: Laboratory Diagnosis. H. W. Whitford, R. F. Rosenbusch, and L. H. Lauerman, ed. Iowa State University Press, Ames.

Untergasser, A., I. Cutcutache, T. Koressaar, J. Ye, B. C. Faircloth, M. Remm, and S. G. Rozen. 2012. Primer3-New capabilities and interfaces. Nucleic Acids Res. 40:e115.

Wawegama, N. K., G. F. Browning, A. Kanci, M. S. Marenda, and P. F. Markham. 2014. Development of a recombinant protein-based enzyme-linked immunosorbent assay for diagnosis of Mycoplasma bovis infection in cattle. Clin. Vaccine Immunol. 21:196-202. https://doi.org/10.1128/CVI.00670-13.

Wawegama, N. K., P. F. Markham, A. Kanci, M. Schibrowski, S. Oswin, T. S. Barnes, S. M. Firestone, T. J. Mahony, and F. Browning. 2016. Evaluation of an IgG enzyme-linked immunosorbent assay as a serological assay for detection of Mycoplasma bovis infection in feedlot cattle. J. Clin. Microbiol. 54:1269-1275. https://doi .org/10.1128/JCM.02492-15. 